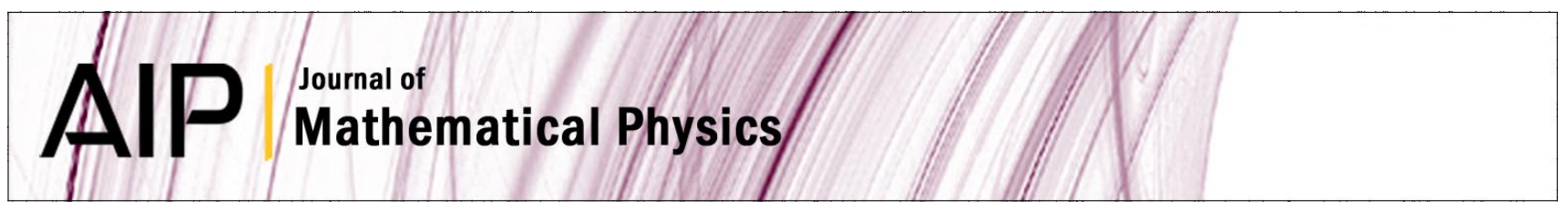

\title{
Stochastic quantization of real-time thermal field theory
}

T. C. de Aguiar, N. F. Svaiter, and G. Menezes

Citation: J. Math. Phys. 51, 102304 (2010); doi: 10.1063/1.3492927

View online: http://dx.doi.org/10.1063/1.3492927

View Table of Contents: http://jmp.aip.org/resource/1/JMAPAQ/v51/i10

Published by the AIP Publishing LLC.

Additional information on J. Math. Phys.

Journal Homepage: http://jmp.aip.org/

Journal Information: http://jmp.aip.org/about/about_the_journal

Top downloads: http://jmp.aip.org/features/most_downloaded

Information for Authors: http://jmp.aip.org/authors

\section{ADVERTISEMENT}

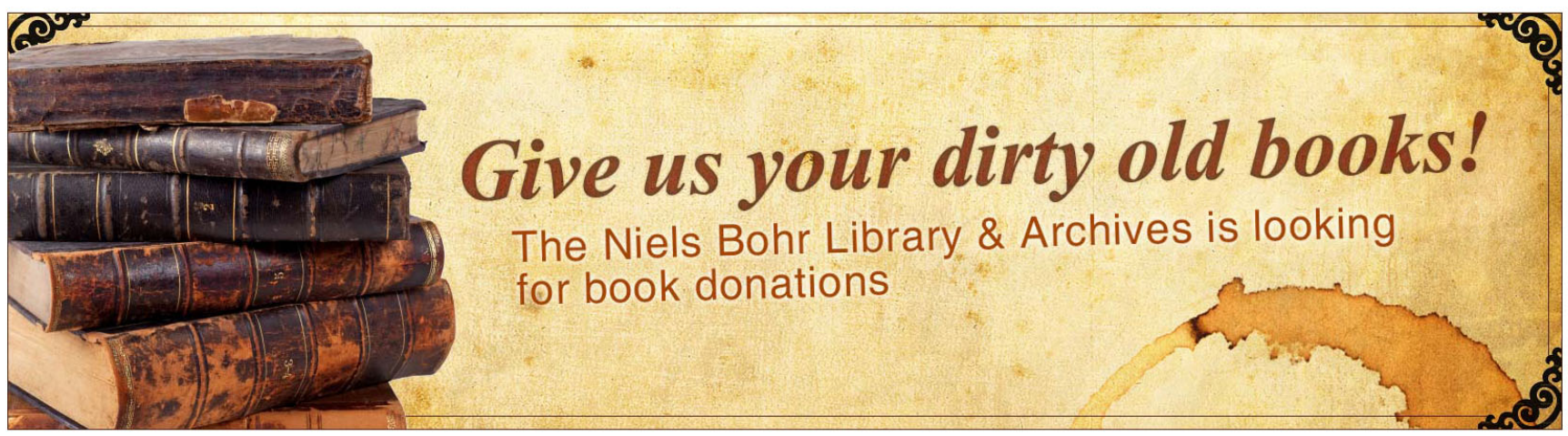




\title{
Stochastic quantization of real-time thermal field theory
}

\author{
T. C. de Aguiar, ${ }^{1, a)}$ N. F. Svaiter, ${ }^{1, b)}$ and G. Menezes ${ }^{2, c)}$ \\ ${ }^{1}$ Centro Brasileiro de Pesquisas Físicas (CBPF), Rua Dr. Xavier Sigaud 150, Rio de \\ Janeiro 22290-180, Rio de Janeiro, Brazil \\ ${ }_{2}^{2}$ Instituto de Física Teórica, Universidade Estadual Paulista, Rua Dr. Bento Teobaldo \\ Ferraz, 271, Bloco II, Barra Funda, São Paulo 01140-070, São Paulo, Brazil
}

(Received 11 May 2010; accepted 2 September 2010; published online 19 October 2010)

\begin{abstract}
We use the stochastic quantization method to obtain the free scalar propagator of a finite temperature field theory formulated in the Minkowski space-time. First, we use the Markovian stochastic quantization approach to present the two-point function of the theory. Second, we assume a Langevin equation with a memory kernel and a colored noise. The convergence of the Markovian and non-Markovian stochastic processes in the asymptotic limit of the fictitious time is obtained. Our formalism can be the starting point to discuss systems at finite temperature out of equilibrium. (C) 2010 American Institute of Physics. [doi:10.1063/1.3492927]
\end{abstract}

\section{INTRODUCTION}

In order to study perturbative quantum field theory at finite temperature, there are three established formalisms: the imaginary time formalism or the Matsubara formalism, ${ }^{1,2}$ the closed time path Green's function formalism, ${ }^{3,4}$ and the thermofield dynamics approach. ${ }^{5,6}$ In the imaginary time formalism, the fundamental idea is to replace the time $t$ by $i t$ in order to obtain the matrix elements of the equilibrium density matrix operator $\exp (-\beta H)$. A perturbative calculation for equilibrium thermal field theory can be developed using this approach. ${ }^{7}$ For nonequilibrium quantum field systems, which must be formulated using the real time, the path ordered method or the thermofield dynamics must be used. The modern field theoretical approach in the closed time path Green's function method, as functional technique and path integral representation, was introduced in the 1980s when it was developed as a unified framework to describe both the equilibrium and the nonequilibrium situations in thermal field theory. Finally, in the thermofield dynamics approach, some degrees of freedom associated with the vacuum in quantum field theory act as thermal degrees of freedom. This thermal field dynamics mechanism for the creation of noise in a pure state can be done by doubling each dynamical degree of freedom of the system. For a complete survey of this method, see, for example, Ref. 8.

An alternative way of quantization is the stochastic quantization method. In this paper, we present an approach to study finite temperature field theory in the real-time formalism using the stochastic quantization method. The Markovian and the non-Markovian stochastic quantization procedures are presented in order to quantize a neutral free scalar field. ${ }^{9}$ The basic ideas of the non-Markovian Langevin equation with a memory kernel can be found in Refs. 10-13.

The program of stochastic quantization, first proposed by Parisi and $\mathrm{Wu},{ }^{14}$ and the stochastic regularization were carried out for systems described by fields defined in flat, Euclidean manifolds. A brief introduction to stochastic quantization can be found in Refs. 15-17 and a complete review is given in Ref. 18. Although the stochastic quantization method was formulated using the Euclidean space-time obtained by Wick's rotation, several authors have attempted to formulate the stochastic quantization directly in Minkowski space-time (see, for example, the discussion pre-

\footnotetext{
${ }^{a)}$ Electronic mail: deaguiar@cbpf.br.

${ }^{b)}$ Electronic mail: nfuxsvai@cbpf.br.

${ }^{c)}$ Electronic mail: gsm@ift.unesp.br.
} 
sented by Hüffel and Rumpf ${ }^{19}$ and Gozzi ${ }^{20}$ ). In the first of these papers, the authors proposed a modification of the original Parisi-Wu scheme by introducing a complex drift term in the Langevin equation in order to implement the stochastic quantization in Minkowski space-time. Gozzi studied the spectrum of the non-self-adjoint Fokker-Planck Hamiltonian to justify this program (see also Refs. 21 and 22). We observe that the implementation of the stochastic quantization in the Minkowski space-time can be seen as a special case of the Euclidean formulation for systems with complex action since the drift term is purely imaginary in this case.

Even when the stochastic quantization is formulated in the Euclidean space-time, difficulties appear in the case of complex actions. A complex action leads to a nonpositive definite measure and the convergence of the stochastic process in the asymptotic limit of the fictitious time is not achieved. There are many examples in literature where Euclidean action is complex. We have, for example, quantum chromodynamics with nonvanishing chemical potential at finite temperature; for $S U(N)$ theories with $N>2$, the fermion determinant becomes complex and also the effective action. Complex terms can also appear in the Langevin equation for fermions, but a suitable kernel, which defines a kerneled Langevin equation, can circumvent this problem. ${ }^{23-25}$ Finally, for topological field theories, the Euclidean action is also complex. The crucial question is the following: what happens if the Langevin equation possesses a complex drift term? Some authors claimed that it is possible to obtain meaningful results out of the Langevin equations describing diffusion processes around a complex action. Parisi ${ }^{26}$ and Klauder and Peterson ${ }^{27}$ investigated the complex Langevin equation, where some numerical simulations in one-dimensional systems were presented (see also Refs. 28 and 29). We would also like to mention the approach developed by Okamoto et al., ${ }^{30}$ where the role of the kernel in the complex Langevin equation was studied. More recently, Guralnik and Pehlevan ${ }^{31}$ constructed an effective potential for the complex Langevin equation on a lattice. These authors also investigated a complex Langevin equation and Dyson-Schwinger equations that appear in such situations. ${ }^{32}$

As we have pointed out, an important case that deserves our attention is the stochastic quantization of topological field theories in the Euclidean formulation. A topological action always contains a factor of $i$ in the Euclidean space-time; therefore, the path integral measure for the topological theory always remains to be $e^{i S}$ even after the Wick rotation. Recently, Menezes and Svaiter ${ }^{33,34}$ implemented the stochastic quantization to study such systems with complex valued path integral weights, where we have a nonpositive definite measure. As mentioned, the convergence of the stochastic process in the asymptotic limit of the fictitious time is not achieved. To circumvent this problem, these authors assumed a Langevin equation with memory kernel and Einstein's relations with colored noise. In the asymptotic limit of the fictitious time, the equilibrium solution of such Langevin equation was analyzed. It was shown that for a large class of elliptic non-Hermitian operators, which define different models in quantum field theory, the solution converges to the correct equilibrium state in the asymptotic limit of the fictitious time $\tau$ $\rightarrow \infty$. In conclusion, in these situations for complex actions in Euclidean field theory, the nonMarkovian approach may be more suitable to deal with nonconvergence problems than the Markovian approach since it is a more general method.

The aim of this paper is to implement the stochastic quantization in the Minkowski spacetime. The theory of real-time Green's functions can be used to describe systems in equilibrium and also out of equilibrium. To include the interaction within this formalism, we can use the results presented in Refs. 9 and 34. In Ref. 9, the non-Markovian stochastic quantization for an interacting scalar field theory formulated in the Euclidean space was presented. Also using Ref. 34, we see that the method proposed can be extended to interacting field theory with complex actions, where a consistent perturbation theory out of equilibrium can be developed.

We would like to point out that recently the stochastic quantization technique, not based in a probability interpretation, was used by Berges and Stamatescu to study the dynamics of an interacting scalar field theory out of equilibrium. ${ }^{35}$ Lattice simulation of a non-Abelian gauge field theory was presented in Ref. 36. As was stressed by many authors, ${ }^{14,37,38}$ the stochastic quantization and the Langevin equation can be extremely useful in numerical simulations of field theory models ${ }^{39,40}$ with complex actions that are difficult to treat nonperturbatively. 
The outline of this paper is the following. The introduction is given in Sec. I. In Sec. II, we present a brief review of the real-time formalism in quantum field theory. In Sec. III, we use the Markovian stochastic quantization method to study a thermal field theory formulated in the Minkowski space-time. The non-Markovian approach of the stochastic quantization applied to a thermal scalar field theory is developed in Sec. IV. Conclusions are given in Sec. V. In the Appendix, convergence conditions for the stochastic process are derived. Summation over repeated indices will always be meant, unless otherwise stated. In this paper, we use $\hbar=c=k_{B}=1$.

\section{REAL-TIME FORMALISM IN FINITE TEMPERATURE QUANTUM FIELD THEORY}

In this section, we give a brief survey of the formulation of field theory at finite temperature in Minkowski space-time. Unlike in the imaginary time formalism, in real-time formulation, sums over Matsubara frequencies are absent and there is no need to analytically extend the Green's functions back to the Minkowski space-time. Moreover, the real-time formalism is the starting point for the development of the nonequilibrium quantum field theory since the investigation of dynamical properties of systems is more naturally performed in this formalism. The real-time formalism can describe nonequilibrium processes because the time variable plays a fundamental role and cannot be traded in for an equilibrium temperature.

For simplicity, we work with a neutral scalar field. The field operator in the Heisenberg picture is given by

$$
\phi(t, \mathbf{x})=e^{i H t} \phi(0, \mathbf{x}) e^{-i H t},
$$

where the time variable $t$ is allowed to be complex. The main quantities to be computed are the thermal Green's functions $G_{C}\left(x_{1}, \ldots, x_{N}\right)$, defined as

$$
G_{C}\left(x_{1}, \ldots, x_{N}\right)=\left\langle T_{C}\left(\phi\left(x_{1}\right) \ldots \phi\left(x_{N}\right)\right)\right\rangle_{\beta},
$$

where the time ordering is taken along a complex time path, yet to be defined. Considering a parametrization $t=z(v)$ of the path, the following expressions:

$$
\begin{gathered}
\theta_{C}\left(t-t^{\prime}\right)=\theta\left(v-v^{\prime}\right), \\
\delta_{C}\left(t-t^{\prime}\right)=\left(\frac{\partial z}{\partial v}\right)^{-1} \delta\left(v-v^{\prime}\right)
\end{gathered}
$$

define the generalized $\theta$ - and $\delta$-functions. The functional differentiation is also extended in the following way:

$$
\frac{\delta j(x)}{\delta j\left(x^{\prime}\right)}=\delta_{C}\left(t-t^{\prime}\right) \delta^{3}\left(\mathbf{x}-\mathbf{x}^{\prime}\right)
$$

for functions $j(x)$ defined on path $C$. The Green's functions defined by Eq. (2) can be obtained from a generating functional $Z_{C}[\beta ; j]$ through the expression

$$
G_{C}\left(x_{1}, \ldots, x_{N}\right)=\left.\frac{(-i)^{N}}{Z_{C}[\beta ; j]} \frac{\delta^{N} Z_{C}[\beta ; j]}{\delta j\left(x_{1}\right) \cdots \delta j\left(x_{N}\right)}\right|_{j=0} .
$$

In the above equation, the generating functional is given by 


$$
\begin{aligned}
Z[\beta ; j] & =\operatorname{Tr}\left[e^{-\beta H} T_{C} \exp \left(i \int_{C} d^{4} x j(x) \phi(x)\right)\right] \\
& =\int \mathcal{D} \phi^{\prime}\left\langle\phi^{\prime}(x) ; t-i \beta\left|T_{C} \exp \left(i \int_{C} d^{4} x j(x) \phi(x)\right)\right| \phi^{\prime}(x) ; t\right\rangle,
\end{aligned}
$$

where path $C$ must go through all the arguments of the Green's functions. It is also possible to note from this expression that path $C$ starts from a time $t_{i}=t$ and ends at a time $t_{f}=t-i \beta$. We may recast the generating functional into the form

$$
Z_{C}[\beta ; j]=\mathcal{N} \exp \left\{-i \int_{C} d^{4} x V\left(\frac{\delta}{i \delta} j(x)\right)\right\} Z_{C}^{F}[\beta ; j],
$$

where $\mathcal{N}$ is a normalization parameter and the free generating functional is given by

$$
Z_{C}^{F}[\beta ; j]=\exp \left\{-\frac{1}{2} \int_{C} d^{4} x \int_{C} d^{4} y j(x) D_{C}^{F}(x-y) j(y)\right\} .
$$

In Eq. (9), the propagator $D_{C}^{F}(x-y)$ is defined through the formula

$$
D_{C}^{F}\left(x-x^{\prime}\right)=\theta_{C}\left(t-t^{\prime}\right) D_{C}^{>}\left(x, x^{\prime}\right)+\theta_{C}\left(t^{\prime}-t\right) D_{C}^{<}\left(x, x^{\prime}\right),
$$

where $D_{C}^{>}\left(x, x^{\prime}\right)$ and $D_{C}^{<}\left(x, x^{\prime}\right)$ are, respectively,

$$
\begin{aligned}
& D_{C}^{>}\left(x, x^{\prime}\right)=\left\langle\phi(x) \phi\left(x^{\prime}\right)\right\rangle_{\beta}, \\
& D_{C}^{<}\left(x, x^{\prime}\right)=\left\langle\phi\left(x^{\prime}\right) \phi(x)\right\rangle_{\beta} .
\end{aligned}
$$

Since the propagator $D_{C}^{F}\left(x-x^{\prime}\right)$ is properly defined in the interval $-\beta \leq \operatorname{Im}\left(t-t^{\prime}\right) \leq \beta$, one may conclude that the path considered must be such that the imaginary part of the time variable $t$ is nonincreasing when the parameter $v$ increases. Furthermore, since we are interested in Green's functions whose arguments are real, path $C$ must contain the real axis. One possible choice for the contour $C$ is described in the following: ${ }^{41}$

(1) $C$ starts from a real value $t_{i}$, large and negative.

(2) The contour follows the real axis up to the large positive value $-t_{i}$. This part of $C$ is denoted by $C_{1}$.

(3) The path from $-t_{i}$ to $-t_{i}-i \frac{\beta}{2}$, along a vertical straight line. This is denoted by $C_{3}$.

(4) The path follows a horizontal line $C_{2}$ going from $-t_{i}-i \frac{\beta}{2}$ to $t_{i}-i \frac{\beta}{2}$.

(5) Finally, the path follows a vertical line $C_{4}$ from $t_{i}-i \frac{\beta}{2}$ to $t_{i}-i \beta$.

Taking $t_{i} \rightarrow-\infty$, the free generating functional can be factorized as

$$
Z_{C}^{F}[\beta ; j]=Z_{C_{1} \cup C_{2}}^{F}[\beta ; j] Z_{C_{3} \cup C_{4}}^{F}[\beta ; j] .
$$

The Green's functions with real-time arguments can be deduced from $Z_{C_{1} \cup C_{2}}^{F}[\beta ; j]$ only. The $Z_{C_{3} \cup C_{4}}^{F}[\beta ; j]$ generating functional can be considered a multiplicative constant. Choosing $t$ and $t^{\prime}$ to be real, running from $-\infty$ to $\infty$, and label the sources $j_{1}(x)=j(t, \mathbf{x})$ and $j_{2}(x)=j(t-i \beta / 2, \mathbf{x})$. Also, one has $\delta j_{a}(x) / \delta j_{b}\left(x^{\prime}\right)=\delta_{a b} \delta^{4}\left(x-x^{\prime}\right)$. With these expressions, one may rewrite the free generating functional as

$$
Z_{C}^{F}[\beta ; j]=\mathcal{N}^{\prime} \exp \left\{-\frac{1}{2} \int d^{4} x \int d^{4} x^{\prime} j_{a}(x) D_{a b}^{F} j_{b}\left(x^{\prime}\right)\right\},
$$

where, again, $\mathcal{N}^{\prime}$ is a normalization parameter. The components of the matricial propagator $D_{a b}^{F}\left(x-x^{\prime}\right)$ are given by 


$$
\begin{gathered}
D_{11}^{F}\left(x-x^{\prime}\right)=D_{F}\left(t-t^{\prime}, \mathbf{x}-\mathbf{x}^{\prime}\right), \\
D_{22}^{F}\left(x-x^{\prime}\right)=D_{F}^{*}\left(t-t^{\prime}, \mathbf{x}-\mathbf{x}^{\prime}\right), \\
D_{12}^{F}\left(x-x^{\prime}\right)=D^{<}\left(t-t^{\prime}+i \beta / 2, \mathbf{x}-\mathbf{x}^{\prime}\right), \\
D_{21}^{F}\left(x-x^{\prime}\right)=D^{>}\left(t-t^{\prime}-i \beta / 2, \mathbf{x}-\mathbf{x}^{\prime}\right) .
\end{gathered}
$$

The effective generating functional can be written as

$$
\begin{aligned}
Z_{C}[\beta ; j]= & \int \mathcal{D} \phi_{1} \mathcal{D} \phi_{2} \exp \left\{-\frac{1}{2} \int d^{4} x d^{4} x^{\prime} \phi_{a}(x)\left(D_{F}^{-1}\right)_{a b}\left(x-x^{\prime}\right) \phi_{b}\left(x^{\prime}\right)\right\} \\
& \times \exp \left\{-i \int d^{4} x\left(V\left(\phi_{1}\right)-V\left(\phi_{2}\right)\right)+i \int d^{4} x j_{a}(x) \phi_{a}(x)\right\} .
\end{aligned}
$$

The field $\phi_{2}$ may be interpreted as a ghost field on the contour $C_{2}$. This doubling of the field degrees of freedom, which does not occur in imaginary time formulation, is unavoidable in the real-time formulation. For more details on this subject, the reader is referred to the original paper of Niemi and Semenoff ${ }^{42}$ or the Landsman and van Weert review. ${ }^{43}$

\section{REAL-TIME FINITE TEMPERATURE QUANTUM FIELD THEORY: THE MARKOVIAN STOCHASTIC QUANTIZATION APPROACH}

The real-time formalism is a framework to describe both equilibrium and nonequilibrium systems. Dynamical questions, as, for example, a weakly interacting Bose gas having a temperature gradient, can be studied only in the real-time formalism, with a matrix structure of the propagator. Before we study the non-Markovian approach, in this section we will analyze the usual stochastic quantization of a finite temperature field theory formulated in the Minkowski space. In the Minkowski space, it is well known that the Langevin equation should be written as

$$
\frac{\partial}{\partial \tau} \phi(x, \tau)=\left.i \frac{\delta S}{\delta \phi(x)}\right|_{\phi(x)=\phi(x, \tau)}+\eta(x, \tau),
$$

where $S(\phi)$ is the action for a free scalar field

$$
S(\phi)=\int d^{4} x \frac{1}{2}\left\{\partial^{\mu} \phi \partial_{\mu} \phi-m^{2}-i \epsilon \phi^{2}\right\},
$$

and the correlation functions for the noise field are

$$
\begin{gathered}
\langle\eta(x, \tau)\rangle_{\eta}=0, \\
\left\langle\eta(x, \tau) \eta\left(x^{\prime}, \tau^{\prime}\right)\right\rangle_{\eta}=2 \delta\left(\left|\tau-\tau^{\prime}\right|\right) \delta^{4}\left(x-x^{\prime}\right) .
\end{gathered}
$$

Owing to the presence of factor $i$ in front of the drift term, the resulting stochastic field $\phi(x, \tau)$ becomes complex valued. Reference 18 emphasizes that the presence of the negative imaginary mass term $-(i / 2) \epsilon \phi^{2}$ in the action given by Eq. (17) is necessary to obtain convergence for the stochastic process being considered. So, it means that we can only take the limit $\epsilon \rightarrow 0$ after all calculations have been performed. It is straightforward to obtain the two-point correlation functions for this case as well as to develop a perturbative solution to the self-interacting situation. Although we do not wish to go into the details here, for the interested reader, we recommend Refs. 19 and 21, where a discussion on stochastic diagrams can be found. 
Now, let us focus our attentions to the finite temperature case in real-time formulation. As we have discussed in Sec. II, the doubling of the field degrees of freedom is unavoidable in the real-time formulation. So, we write the field as an isovector $\phi=\left(\begin{array}{c}\phi_{1} \\ \phi_{2}\end{array}\right)$. The action for the isovector scalar field, in the free case, is given by

$$
S=\frac{1}{2} \int d^{4} x d^{4} x^{\prime} \phi_{a}(x)\left(D_{F}^{-1}\right)_{a b}\left(x-x^{\prime}\right) \phi_{b}\left(x^{\prime}\right),
$$

where the components of $D_{F}$ are given by Eq. (14). In the Fourier space,

$$
\left(D_{F}\right)_{a b}(k)=\left(U^{t}\right)_{a c}(\theta)\left(D_{0}\right)_{c d}(k)(U)_{d b}(\theta)
$$

where

$$
U(\theta)=\left(\begin{array}{cc}
\cosh \theta & \sinh \theta \\
\sinh \theta & \cosh \theta
\end{array}\right)
$$

with

$$
\cosh ^{2} \theta=\frac{e^{\beta\left|k_{0}\right|}}{e^{\beta\left|k_{0}\right|}-1}
$$

and $D_{0}$ is given by

$$
D_{0}(k)=\left(\begin{array}{cc}
\frac{1}{k^{2}-m^{2}+i \epsilon} & 0 \\
0 & \frac{-1}{k^{2}-m^{2}-i \epsilon}
\end{array}\right) .
$$

So, we can split $D_{F}$ into two parts, $D_{F}(k)=D_{0}(k)+D_{\beta}(k)$, where $D_{0}(k)$ is temperature independent and all temperature dependence appears in $D_{\beta}(k)$, which is given by

$$
D_{\beta}(k)=\frac{-i \epsilon}{\left(k^{2}-m^{2}\right)^{2}+\epsilon^{2}}\left(\begin{array}{cc}
2 \sinh ^{2} \theta & \sinh 2 \theta \\
\sinh 2 \theta & 2 \sinh ^{2} \theta
\end{array}\right)
$$

Notice that in the limit $\epsilon \rightarrow 0$, we have

$$
\frac{\epsilon}{\left(k^{2}-m^{2}\right)^{2}+\epsilon^{2}} \rightarrow \pi \delta\left(k^{2}-m^{2}\right)
$$

As in the zero temperature case, we have to use the above expressions with finite $\epsilon$ and take the $\epsilon \rightarrow 0$ limit after all the calculations have been done in order to obtain convergence in the limit $\tau \rightarrow \infty$. We also know that this is the case for the path integral formalism. ${ }^{42}$ The Markovian Langevin equation for the nonequilibrium case is given by

$$
\frac{\partial}{\partial \tau} \phi_{a}(k, \tau)=i\left(D_{F}^{-1}\right)_{a b}(k) \phi_{b}(k, \tau)+\eta_{a}(k, \tau),
$$

where $\phi=\left(\begin{array}{l}\phi_{1} \\ \phi_{2}\end{array}\right), a, b=1,2$, and from Eqs. (24) and (25), 


$$
\begin{aligned}
D_{F}^{-1}(k)= & I(k, \epsilon) \\
& \times\left(\begin{array}{ll}
\frac{-1}{k^{2}-m^{2}-i \epsilon}-\frac{i \epsilon}{\left(k^{2}-m^{2}\right)^{2}+\epsilon^{2}} 2 \sinh ^{2} \theta & \frac{i \epsilon}{\left(k^{2}-m^{2}\right)^{2}+\epsilon^{2}} \sinh 2 \theta \\
\frac{i \epsilon}{\left(k^{2}-m^{2}\right)^{2}+\epsilon^{2}} \sinh 2 \theta & \frac{1}{k^{2}-m^{2}+i \epsilon}-\frac{i \epsilon}{\left(k^{2}-m^{2}\right)^{2}+\epsilon^{2}} 2 \sinh ^{2} \theta
\end{array}\right),
\end{aligned}
$$

where

$$
I(k, \epsilon)=\frac{-\left(k^{2}-m^{2}\right)^{2}-\epsilon^{2}\left(\cosh ^{4} \theta+\sinh ^{4} \theta\right)-\frac{\epsilon^{2}}{2} \sinh ^{2} 2 \theta}{\left(\left(k^{2}-m^{2}\right)^{2}+\epsilon^{2}\right)} .
$$

The noise correlation functions are given by

$$
\begin{gathered}
\left\langle\eta_{a}(k, \tau)\right\rangle_{\eta}=0, \\
\left\langle\eta_{a}(k, \tau) \eta_{b}\left(k^{\prime}, \tau^{\prime}\right)\right\rangle_{\eta}=2(2 \pi)^{4} \delta_{a b} \delta^{4}\left(k+k^{\prime}\right) \delta\left(\left|\tau-\tau^{\prime}\right|\right),
\end{gathered}
$$

and, again, $\eta=\left(\begin{array}{l}\eta_{1} \\ \eta_{2}\end{array}\right), a, b=1,2$. Here, we emphasize that since the drift term in the Langevin equation (27) is a complex quantity, each component $\phi_{a}(k, \tau)$ becomes complex, $\phi_{a}=\phi_{a R}+i \phi_{a I}$. Maintaining a real noise, we obtain two Langevin equations,

$$
\frac{\partial}{\partial \tau} \phi_{a R}(k, \tau)=-\operatorname{Re}\left[\left(D_{F}^{-1}\right)_{a b}(k)\right] \phi_{b I}(k, \tau)-\operatorname{Im}\left[\left(D_{F}^{-1}\right)_{a b}(k)\right] \phi_{b R}(k, \tau)+\eta_{a}(k, \tau)
$$

and

$$
\frac{\partial}{\partial \tau} \phi_{a I}(k, \tau)=\operatorname{Re}\left[\left(D_{F}^{-1}\right)_{a b}(k)\right] \phi_{b R}(k, \tau)-\operatorname{Im}\left[\left(D_{F}^{-1}\right)_{a b}(k)\right] \phi_{b I}(k, \tau) .
$$

This separation into real and imaginary parts will be important when we discuss the FokkerPlanck approach. However, for the moment we will treat it as a single equation for the complex field $\phi_{a}$, i.e., we treat Eq. (27) as a holomorphic one. So, its solution will be given by

$$
\phi_{a}(k, \tau)=\int^{\infty} d \tau^{\prime} g_{a b}\left(k, \tau-\tau^{\prime}\right) \eta_{b}\left(k, \tau^{\prime}\right),
$$

where $g(k, \tau)=e^{i D_{F}^{-1}(k) \tau} \theta(\tau)$ is the Green's function for the diffusion problem.

Now, we should worry ourselves about the convergence of this stochastic process, i.e., we should analyze if $\left.g(k, \tau)\right|_{\tau \rightarrow \infty} \rightarrow 0$. In order to do so, we must first diagonalize the matrix $i D_{F}^{-1}(k)$. Doing such calculation, we get the matrix $D^{\prime}(k)$, given by

$$
D^{\prime}(k)=i I(k, \epsilon)\left(\begin{array}{cc}
\lambda_{+} & 0 \\
0 & \lambda_{-}
\end{array}\right)
$$

where

$$
\lambda_{ \pm}=\frac{ \pm \sqrt{\left(k^{2}-m^{2}\right)^{2}-\epsilon^{2} \sinh ^{2} 2 \theta}-i \epsilon\left(1+2 \sinh ^{2} \theta\right)}{\left(k^{2}-m^{2}\right)^{2}+\epsilon^{2}} .
$$

Since $I(k, \epsilon)<0$, we notice from the above equations that, indeed, we get $\left.g(k, \tau)\right|_{\tau \rightarrow \infty} \rightarrow 0$. We also remark that as in the zero temperature case, the convergence of the stochastic process was possible because we have maintained in Eqs. (24) and (25) a finite $\epsilon$. As the reader can easily verify from 
Eqs. (35) and (36), if we take the $\epsilon \rightarrow 0$ limit in the beginning of the calculations, we should lose the convergence factor $e^{-I(k, \epsilon) \epsilon\left(1+2 \sinh ^{2} \theta\right)}$. So, similar to the path integral formalism mentioned above, this limit should be taken after all the calculations have been done. In doing so, it is easy to see that we obtain convergence in the limit $\tau \rightarrow \infty$.

Now, we are ready to calculate the two-point function $\left\langle\phi_{a}(k, \tau) \phi_{b}\left(k^{\prime}, \tau\right)\right\rangle_{\eta}$. Proceeding with similar calculations as in the zero temperature case, it is possible to show that the two-point correlation function is given by

$$
\left\langle\phi_{a}(k, \tau) \phi_{b}\left(k^{\prime}, \tau\right)\right\rangle_{\eta}=(2 \pi)^{4} \delta^{4}\left(k+k^{\prime}\right) i\left(D_{F}\right)_{a c}(k)\left(1-e^{2 i D_{F}^{-1}(k) \tau}\right)_{c b},
$$

so we see that in the limit $\tau \rightarrow \infty$, we recover the usual result. We are interested now to see the effects of a memory kernel in this nonequilibrium quantum field theory. This is the subject of Sec. IV.

\section{REAL-TIME FINITE TEMPERATURE QUANTUM FIELD THEORY: THE NON- MARKOVIAN STOCHASTIC QUANTIZATION APPROACH}

The aim of this section is to study finite temperature quantum field theory in the Minkowski space-time using the non-Markovian stochastic quantization approach. In the Minkowski spacetime, the Langevin equation with memory kernel is written as

$$
\frac{\partial}{\partial \tau} \phi(x, \tau)=\left.i \int_{0}^{\tau} d s M_{\Lambda}(\tau-s) \frac{\delta S}{\delta \phi(x)}\right|_{\phi(x)=\phi(x, s)}+\eta(x, \tau),
$$

where $S$ is the action for the free scalar field, given by Eq. (20). The noise field distribution is such that its first and second momenta are given by

$$
\begin{gathered}
\left\langle\eta_{a}(x, \tau)\right\rangle_{\eta}=0, \\
\left\langle\eta_{a}(x, \tau) \eta_{b}\left(x^{\prime}, \tau^{\prime}\right)\right\rangle_{\eta}=2 \delta_{a b} M_{\Lambda}\left(\left|\tau-\tau^{\prime}\right|\right) \delta^{4}\left(x-x^{\prime}\right),
\end{gathered}
$$

that is, we have a colored Gaussian distribution. We remind the reader that Eq. (38) is to be understood as a matrix equation. Also, since the drift term in this non-Markovian Langevin equation is complex again, we have the same separation as before, $\phi_{a}=\phi_{a R}+i \phi_{a I}$ and we keep the noise as a real quantity.

Using a Fourier decomposition for the scalar and noise fields, given by

$$
X(x, \tau)=\frac{1}{(2 \pi)^{2}} \int d^{4} k e^{i k x} X(k, \tau),
$$

where the field $X$ represents either the noise field $\eta$ or the scalar field $\phi$, we obtain that each Fourier mode $\phi(k, \tau)$ satisfies a Langevin equation of the form

$$
\frac{\partial}{\partial \tau} \phi_{a}(k, \tau)=i \int_{0}^{\tau} d s M_{\Lambda}(\tau-s)\left(D_{F}^{-1}\right)_{a b}(k) \phi_{b}(k, s)+\eta_{a}(k, \tau)
$$

where $D_{F}^{-1}(k)$ is the inverse of $D_{F}(k)$, defined by Eq. (21). With this decomposition, we obtain the following relations for the noise field Fourier components from Eqs. (39) and (40):

$$
\begin{gathered}
\left\langle\eta_{a}(k, \tau)\right\rangle_{\eta}=0, \\
\left\langle\eta_{a}(k, \tau) \eta_{b}\left(k^{\prime}, \tau^{\prime}\right)\right\rangle_{\eta}=2(2 \pi)^{4} \delta_{a b} M_{\Lambda}\left(\left|\tau-\tau^{\prime}\right|\right) \delta^{4}\left(k+k^{\prime}\right) .
\end{gathered}
$$

Defining the Laplace transform of the memory kernel as 


$$
M(z)=\int_{0}^{\infty} d \tau M_{\Lambda}(\tau) e^{-z \tau}
$$

we obtain the solution for Eq. (42), subject to the initial condition $\phi_{a}(k, \tau=0)=0, a=1,2$,

$$
\phi_{a}(k, \tau)=\int_{0}^{\infty} d \tau^{\prime} G_{a b}\left(k, \tau-\tau^{\prime}\right) \eta_{b}\left(k, \tau^{\prime}\right)
$$

In Eq. (46), $G_{a b}\left(k, \tau-\tau^{\prime}\right)=\Omega_{a b}\left(k, \tau-\tau^{\prime}\right) \theta\left(\tau-\tau^{\prime}\right)$ is the retarded Green's function for this nonMarkovian diffusion problem and each component of the $\Omega$-matrix is defined through its Laplace transform,

$$
\Omega_{a b}=\left[z \mathcal{I}-i M(z) D_{F}^{-1}(k)\right]_{a b}^{-1},
$$

where $\mathcal{I}$ denotes a $2 \times 2$ identity matrix. Thus, the two-point correlation function in the Fourier representation is written as

$$
\begin{aligned}
\left\langle\phi_{a}(k, \tau) \phi_{b}\left(k^{\prime}, \tau^{\prime}\right)\right\rangle_{\eta} & =D_{a b}\left(k, \tau, \tau^{\prime}\right) \\
& =(2 \pi)^{4} \delta^{4}\left(k+k^{\prime}\right) \int_{0}^{\tau} d s \int_{0}^{\tau^{\prime}} d s^{\prime}\left[\Omega\left(k, \tau-\tau^{\prime}\right) \Omega\left(k, \tau-s^{\prime}\right)\right]_{a b} M_{\Lambda}\left(\left|s-s^{\prime}\right|\right) .
\end{aligned}
$$

The two-dimensional Laplace transform of the above equation is given by

$$
\begin{aligned}
& \int_{0}^{\infty} d \tau e^{-z \tau} \int_{0}^{\infty} d \tau^{\prime} e^{-z^{\prime} \tau^{\prime}} \int_{0}^{\tau} d s \int_{0}^{\tau^{\prime}} d s^{\prime}\left[\Omega\left(k, \tau-\tau^{\prime}\right) \Omega\left(k, \tau-s^{\prime}\right)\right]_{a b} M_{\Lambda}\left(\left|s-s^{\prime}\right|\right) \\
& \quad=\left[\Omega(k, z) \Omega\left(k^{\prime}, z^{\prime}\right)\right]_{a b}\left(\frac{M(z)+M\left(z^{\prime}\right)}{z+z^{\prime}}\right) .
\end{aligned}
$$

Using Eq. (47), this expression becomes

$$
\begin{aligned}
& \int_{0}^{\infty} d \tau e^{-z \tau} \int_{0}^{\infty} d \tau^{\prime} e^{-z^{\prime} \tau^{\prime}} \int_{0}^{\tau} d s \int_{0}^{\tau^{\prime}} d s^{\prime}\left[\Omega\left(k, \tau-\tau^{\prime}\right) \Omega\left(k, \tau-s^{\prime}\right)\right]_{a b} M_{\Lambda}\left(\left|s-s^{\prime}\right|\right) \\
& =i\left(\frac{\Omega(k, z)+\Omega\left(k, z^{\prime}\right)}{z+z^{\prime}}-\Omega(k, z) \Omega\left(k, z^{\prime}\right)\right)_{a c}\left(D_{F}\right)_{c b}(k) .
\end{aligned}
$$

Applying the inverse transform, we obtain for the two-point function

$$
D_{a b}\left(k, \tau, \tau^{\prime}\right)=2 i(2 \pi)^{4} \delta^{4}\left(k+k^{\prime}\right)\left(\Omega\left(k,\left|\tau^{\prime}-\tau\right|\right)-\Omega(k, \tau) \Omega\left(k, \tau^{\prime}\right)\right)_{a c}\left(D_{F}\right)_{c b}(k) .
$$

In order to investigate the convergence of the above equation, we need to specify an expression for the memory kernel $M_{\Lambda}$. We set

$$
M_{\Lambda}(\tau)=\frac{1}{2} \Lambda^{2} e^{-\Lambda^{2}|\tau|}
$$

Substituting the Laplace transform of Eq. (52) into Eq. (47), we have that the $\Omega$-matrix is given by

$$
\Omega(k, \tau)=\left(\begin{array}{ll}
\Omega_{11}(k, \tau) & \Omega_{12}(k, \tau) \\
\Omega_{21}(k, \tau) & \Omega_{22}(k, \tau)
\end{array}\right)
$$

where the components $\Omega_{a b}(k, \tau)$ are given in the Appendix. So, we are in a position to present an expression for the two-point correlation function in the limit $\tau=\tau^{\prime} \rightarrow \infty$, 


$$
\left.D_{a b}\left(k, \tau, \tau^{\prime}\right)\right|_{\tau=\tau^{\prime} \rightarrow \infty}=i(2 \pi)^{4} \delta^{4}\left(k+k^{\prime}\right)\left(D_{F}\right)_{a b}(k)
$$

so that, in the limit $\epsilon \rightarrow 0$, we have

$$
\left.D_{a b}\left(k, \tau, \tau^{\prime}\right)\right|_{\tau=\tau^{\prime} \rightarrow \infty ; \epsilon \rightarrow 0}=\left.i(2 \pi)^{4} \delta^{4}\left(k+k^{\prime}\right)\left(D_{F}\right)_{a b}(k)\right|_{\epsilon \rightarrow 0} .
$$

A question still remains opened. What are, if any, the advantages of our non-Markovian method over the usual Markovian one? In order to answer such question, we shall apply a Fokker-Planck analysis. As we know from Euclidean stochastic quantization, correlation functions are introduced as averages over $\eta$,

$$
\begin{aligned}
& \left\langle\phi\left(x_{1}, \tau_{1}\right) \phi\left(x_{2}, \tau_{2}\right) \cdots \phi\left(x_{n}, \tau_{n}\right)\right\rangle_{\eta} \\
& \quad=N^{-1} \int[d \eta] \exp \left(-\frac{1}{4} \int d^{4} x \int d \tau \eta^{2}(x, \tau)\right) \phi\left(x_{1}, \tau_{1}\right) \phi\left(x_{2}, \tau_{2}\right) \cdots \phi\left(x_{n}, \tau_{n}\right),
\end{aligned}
$$

where $\phi$ obeys an Euclidean Langevin equation

$$
\frac{\partial}{\partial \tau} \phi(x, \tau)=-\left.\frac{\delta S}{\delta \phi(x)}\right|_{\phi(x)=\phi(x, \tau)}+\eta(x, \tau),
$$

and $S$ is the Euclidean version of the action given by Eq. (17). $N$ is given by

$$
N=\int[d \eta] \exp \left(-\frac{1}{4} \int d^{4} x \int d \tau \eta^{2}(x, \tau)\right) .
$$

An alternative way to write this average is to introduce the probability density $P[\phi, \tau]$, which is defined as ${ }^{44}$

$$
P[\phi, \tau] \equiv \int[d \eta] \exp \left(-\frac{1}{4} \int d^{4} x \int d \tau \eta^{2}(x, \tau)\right) \prod_{y} \delta(\phi(y)-\phi(y, \tau)) .
$$

In terms of $P$, the correlation functions will read as

$$
\left\langle\phi\left(x_{1}, \tau_{1}\right) \phi\left(x_{2}, \tau_{2}\right) \cdots \phi\left(x_{n}, \tau_{n}\right)\right\rangle_{\eta}=N \int[d \phi] \phi\left(x_{1}\right) \phi\left(x_{2}\right) \cdots \phi\left(x_{n}\right) P[\phi, \tau] .
$$

The free probability density $P$ satisfies the following Fokker-Planck equation:

$$
\frac{\partial}{\partial \tau} P[\phi, \tau]=\int d^{4} x \frac{\delta}{\delta \phi(x)}\left(\frac{\delta}{\delta \phi(x)}+\frac{\delta S}{\delta \phi(x)}\right) P[\phi, \tau],
$$

with the initial condition

$$
P[\phi, 0]=\prod_{y} \delta(\phi(y)) .
$$

The stochastic quantization says that we shall have

$$
w . \lim _{\tau \rightarrow \infty} P[\phi, \tau]=\frac{\exp (-S[\phi])}{\int[d \phi] \exp (-S[\phi])},
$$

where the limit is supposed to be taken "weakly" in the sense of Ref. 44.

In the case that the drift term of the Langevin equation is a complex quantity, much more care is needed if one is trying to calculate the corresponding Fokker-Planck equation. When $\phi$ obeys Eq. (16), i.e., we are in the Minkowski space, Ref. 45 shows us that it is possible to obtain a 
corresponding Fokker-Planck equation. For the finite temperature real-time case, since we also have double field degrees of freedom, we have similar equations as the case worked out above. First, any stochastic expectation value can be written as

$$
\left\langle F\left[\phi_{1}(x, \tau), \phi_{2}(x, \tau)\right]\right\rangle_{\eta}=N^{\prime \prime} \int \prod_{i=1}^{2}\left[d \eta_{i}\right] \exp \left(-\frac{1}{2} \int d^{4} x \int d \tau \eta_{i}(x, \tau) \delta_{i j} \eta_{j}(x, \tau)\right) F\left[\phi_{1}(x, \tau), \phi_{2}(x, \tau)\right],
$$

where each component $\phi_{a}(x, \tau), a=1,2$, obeys Eq. (27) and

$$
N^{\prime \prime-1}=\int \prod_{i=1}^{2}\left[d \eta_{i}\right] \exp \left(-\frac{1}{2} \int d^{4} x \int d \tau \eta_{i}(x, \tau) \delta_{i j} \eta_{j}(x, \tau)\right)
$$

An usual procedure shows us that the distributional functional $P\left[\phi_{a}, \tau\right]$ obeys the following Fokker-Planck equation in momentum space:

$$
\begin{aligned}
\frac{\partial}{\partial \tau} P= & \int d^{4} k\left[\frac{\delta^{2} P}{\delta \phi_{a R}(k) \delta \phi_{a R}(-k)}\right. \\
& +\frac{\delta}{\delta \phi_{a R}(k)}\left(\left(\operatorname{Re}\left[\left(D_{F}^{-1}\right)_{a b}(k)\right] \phi_{b I}(k)+\operatorname{Im}\left[\left(D_{F}^{-1}\right)_{a b}(k)\right] \phi_{b R}(k)\right) P\right) \\
& \left.+\frac{\delta}{\delta \phi_{a I}(k)}\left(\left(-\operatorname{Re}\left[\left(D_{F}^{-1}\right)_{a b}(k)\right] \phi_{b R}(k)+\operatorname{Im}\left[\left(D_{F}^{-1}\right)_{a b}(k)\right] \phi_{b I}(k)\right) P\right)\right],
\end{aligned}
$$

with $P=P\left[\phi_{a}, \tau\right]$. Following similar steps as in Ref. 45, we may get the relations

$$
\begin{aligned}
\left.P\left[\phi_{1}, \phi_{2}, \tau\right]\right|_{\tau \rightarrow \infty}= & N_{e q}^{-1} \exp \left(-\int d^{4} k \phi_{a R}(k) \widetilde{I}_{a b} \phi_{b R}(-k)\right) \\
& \times \exp \left(\int d ^ { 4 } k \left(\phi_{a I}(k)\left(\widetilde{I}+\widetilde{I}^{3} \cdot\left(\widetilde{I}^{2}+\widetilde{R}^{2}\right)^{-1}\right)_{a b} \phi_{b I}(-k)\right.\right. \\
& \left.\left.-2 \phi_{a R}(k)\left(\widetilde{I}^{2} \cdot \widetilde{R}^{-1}\right)_{a b} \phi_{b I}(-k)\right)\right)
\end{aligned}
$$

where $\widetilde{R}=\operatorname{Re}\left[i\left(D_{F}^{-1}\right)_{a b}(k)\right], \widetilde{I}=\operatorname{Im}\left[i\left(D_{F}^{-1}\right)_{a b}(k)\right],\left(D_{F}^{-1}\right)_{a b}(k)$ is the inverse of the $\left(D_{F}\right)_{a b}(k)$, given by Eq. (21), and

$$
\left.\frac{\partial P\left[\phi_{1}, \phi_{2}, \tau\right]}{\partial \tau}\right|_{\tau \rightarrow \infty} \rightarrow \mathrm{O}\left(e^{-I(k, \epsilon) \epsilon\left(1+2 \sinh ^{2} \theta\right) \tau}\right)
$$

In resemblance to the results found in Ref. 45, in spite of the fact that the equilibrium distribution given by Eq. (67) is not of the form $e^{i S}$, it can be proved that the expectation values over this real probability distribution coincide with those obtained by the usual path integral formalism. Besides that, we see that the convergence factor found in Eq. (68) depends on $k$ values, so that it cannot be put outside the $k$ integral that appears when one is calculating $\partial P[\phi, \tau] / \partial \tau$. So, in principle, the convergence in the limit $\tau \rightarrow \infty$ is a bit more cumbersome for this case.

In our real-time non-Markovian case, we notice the similarity between our retarded Green's function $G_{a b}(k, \tau)$ and the one found in Ref. 9, we may follow similar steps to calculate the free probability density. For instance, the free Fokker-Planck equation is 


$$
\begin{aligned}
\frac{\partial}{\partial \tau} P= & \int d^{4} k\left[\frac{\delta^{2}}{\delta \phi_{a R}(k) \delta \phi_{b R}(-k)} K_{a b}(k, \tau) P-\frac{\delta}{\delta \phi_{a R}(k)}\left(\operatorname{Re}\left(i \frac{\delta N}{\delta \phi_{a R}(k)}\right) P\right)+\right. \\
& \left.-\frac{\delta}{\delta \phi_{a I}(k)}\left(\operatorname{Im}\left(i \frac{\delta N}{\delta \phi_{a I}(k)}\right) P\right)\right],
\end{aligned}
$$

where

$$
K_{a b}(k, \tau-s) \equiv 2 \int_{0}^{\tau} d s M_{\Lambda}(\tau-s) G_{a b}(k, \tau-s),
$$

with $G_{a b}(k, \tau-s)$ being the retarded Green's function for the non-Markovian diffusion problem, and

$$
N=N[\phi ; k, \tau-s] \equiv \int_{0}^{\tau} d s M_{\Lambda}(\tau-s) S(\phi(k)),
$$

with $S$ being the Fourier transform of Eq. (20). Again, following similar steps as those in Refs. 9 and 45 , it is easy to verify that an equilibrium solution to this non-Markovian Fokker-Planck equation will be of a similar form of Eq. (67), apart from some possible unimportant constants. Therefore, the probability distribution $P[\phi, \tau]$ will satisfy, up to constants, similar relations as obtained above. However, in our approach, the convergence factor will always be given by $e^{-\Lambda^{2} \tau}$, which does not depend on $k$ or $\epsilon$ values (remember that we consider $\epsilon \rightarrow 0$, even though this limit is only taken at the end of the calculations). It is worth remarking that even though the drift term in the Langevin equation is complex, the probability distribution obtained is real, and the complex character appears only in the field. Since $\Lambda$ is a free parameter of the model, it can always be adjusted so that we may get an improved convergence. Of course, these situations would be best analyzed in a numerical simulation, which is outside the scope of the present work.

\section{CONCLUSIONS}

To quantize a classical thermal field theory out of equilibrium using the stochastic quantization, we have to work in the Minkowski space-time, where, naturally, an imaginary drift term appears in the Langevin equation, which leads to problems with convergence of the stochastic process. The problems of nonconvergence of the Langevin equation in the stochastic quantization framework also appears if someone considers the stochastic quantization of classical fields defined in a generic curved manifold. For curved static manifolds, the implementation of the stochastic quantization is straightforward. ${ }^{46,47}$ Nevertheless, for nonstatic curved manifolds, we have to extend the formalism beyond the Euclidean signature, i.e., to formulate the stochastic quantization in pseudo-Riemannian manifold, instead of formulating it in the Riemannian space, as was originally proposed.

The main difference between the implementation of the stochastic quantization in the Minkowski space-time and in the Euclidean space-time is the fact that in the latter case the approach to the equilibrium state is a stationary solution of the Fokker-Planck equation. In the Minkowski formulation, the Hamiltonian is non-Hermitian and the eigenvalues of such Hamiltonian are in general complex. The real part of such eigenvalues is important to the asymptotic behavior at large Markov time, and the approach to the equilibrium is achieved only if we can show its positive semidefiniteness.

In this paper, we use the stochastic quantization to study thermal field theory formulated in real time. First, we use the Markovian stochastic quantization approach to present the two-point function of the theory. Second, we assumed a Langevin equation with a memory kernel and Einstein's relation with colored noise. The equilibrium solution of such Langevin equation was analyzed. We have shown that the stochastic process converges in the asymptotic limit of the fictitious time $\tau \rightarrow \infty$ and we have obtained the free Green's functions of the theory. Our approach 
based on stochastic quantization using a non-Markovian Langevin equation proved to be well suited to quantize a classical free field out of equilibrium in the real-time formalism at finite temperature.

Although nontrivial, the method can be extended to interacting field theory where a consistent perturbation theory out of equilibrium can be developed. Being more precise, the reader may recall that for weakly coupled fields, the interacting theory is obtained by a perturbation series over the free Gaussian theory. In this situation, the Green's function for the diffusion problem that is used to solve the Langevin equation is the same as the one in the Langevin equation for the noninteracting field theory. Since the convergence in the limit of the fictitious time $\tau \rightarrow \infty$ is analyzed through this Green's function, we notice that the convergence criterion for the interacting case should be the same as the free case. Therefore, apart from some tedious algebra manipulations, the extension of our method to self-interacting theories may be straightforward even though it may not be a trivial task. The implementation of this non-Markovian Langevin equation to study interacting field theory out of equilibrium is under investigation by the authors.

\section{ACKNOWLEDGMENTS}

We would like to thank Gastão Krein for many helpful discussions. N. F. Svaiter would like to acknowledge the hospitality of the Instituto de Física Teórica, Universidade Estadual Paulista, where part of this paper was carried out. This paper was supported by Conselho Nacional de Desenvolvimento Cientifico e Tecnológico do Brazil (CNPq) and Fundação de Amparo a Pesquisa de São Paulo (FAPESP).

\section{APPENDIX: Calculation of components $\Omega$-matrix}

In this appendix, we derive the components $\Omega_{a b}(k, \tau)$. We can write the $\Omega$-matrix as an inverse of matrix $A$, whose components are given by

$$
A=\left(\begin{array}{ll}
z-i M(z) d^{\prime} & i M(z) b^{\prime} \\
i M(z) b^{\prime} & z-i M(z) a^{\prime}
\end{array}\right) .
$$

The quantities that appear in the $A$-matrix are defined by

$$
\begin{aligned}
& a^{\prime}=\frac{a}{a d-b^{2}}, \\
& b^{\prime}=\frac{b}{a d-b^{2}}, \\
& d^{\prime}=\frac{d}{a d-b^{2}},
\end{aligned}
$$

and

$$
\begin{gathered}
a=\frac{1}{k^{2}-m^{2}+i \epsilon}-\frac{i \epsilon}{\left(k^{2}-m^{2}\right)^{2}+\epsilon^{2}} 2 \sinh ^{2} \theta, \\
b=\frac{-i \epsilon}{\left(k^{2}-m^{2}\right)^{2}+\epsilon^{2}} \sinh 2 \theta,
\end{gathered}
$$




$$
d=\frac{-1}{k^{2}-m^{2}-i \epsilon}-\frac{i \epsilon}{\left(k^{2}-m^{2}\right)^{2}+\epsilon^{2}} 2 \sinh ^{2} \theta .
$$

So, we will have

$$
(\Omega)_{a b}(k, z)=\left(A^{-1}\right)_{a b}=\left(\begin{array}{ll}
\Omega_{11}(k, z) & \Omega_{12}(k, z) \\
\Omega_{21}(k, z) & \Omega_{22}(k, z)
\end{array}\right),
$$

where

$$
\begin{gathered}
\Omega_{11}(k, z)=\frac{z-i M(z) a^{\prime}}{\left(z-i M(z) a^{\prime}\right)\left(z-i M(z) d^{\prime}\right)+M^{2}(z) b^{\prime 2}}, \\
\Omega_{12}(k, z)=\Omega_{21}(z)=\frac{-i M(z) b^{\prime}}{\left(z-i M(z) a^{\prime}\right)\left(z-i M(z) d^{\prime}\right)+M^{2}(z) b^{\prime 2}}, \\
\Omega_{22}(k, z)=\frac{z-i M(z) d^{\prime}}{\left(z-i M(z) a^{\prime}\right)\left(z-i M(z) d^{\prime}\right)+M^{2}(z) b^{\prime 2}} .
\end{gathered}
$$

The Laplace transform for the memory kernel [Eq. (52)] is given by

$$
M(z)=\frac{\Lambda^{2}}{2} \frac{1}{z+\Lambda^{2}} .
$$

So, inserting this result into Eqs. (A9)-(A11), we get

$$
\begin{gathered}
\Omega_{11}(k, z)=\frac{P\left(z, t_{a}\right)}{Q(z)}, \\
\Omega_{12}(k, z)=\Omega_{21}(z)=\frac{-\left(t_{b} z+t_{b} \Lambda^{2}\right)}{Q(z)}, \\
\Omega_{22}(k, z)=\frac{P\left(z, t_{d}\right)}{Q(z)},
\end{gathered}
$$

where $t_{j}=i\left(j^{\prime} \Lambda^{2} / 2\right), j=a, b, d$, and

$$
\begin{gathered}
P\left(z, t_{j}\right)=z^{3}+2 \Lambda^{2} z^{2}+\left(\Lambda^{4}-t_{j}\right) z-t_{j} \Lambda^{2}, \\
Q(z)=z^{4}+2 \Lambda^{2} z^{3}+\left(\Lambda^{4}-u\right) z^{2}-u \Lambda^{2} z+v,
\end{gathered}
$$

with $u=i\left(a^{\prime}+d^{\prime}\right) \Lambda^{2} / 2$ and $v=\left(b^{\prime 2}-a^{\prime} d^{\prime}\right) \Lambda^{4} / 4$. From Eqs. (A2)-(A4), we have that

$$
u=\frac{\Lambda^{2} \epsilon\left(1+2 \sinh ^{2} \theta\right)\left(\left(k^{2}-m^{2}\right)^{2}+\epsilon^{2}\right)}{-\left(k^{2}-m^{2}\right)^{2}-\epsilon^{2}\left(\cosh ^{4} \theta+\sinh ^{4} \theta\right)+\frac{\epsilon^{2}}{2} \sinh ^{2} 2 \theta}
$$

and 


$$
v=-\frac{\Lambda^{4}}{4} \frac{\left(\left(k^{2}-m^{2}\right)^{2}+\epsilon^{2}\right)}{-\left(k^{2}-m^{2}\right)^{2}-\epsilon^{2}\left(\cosh ^{4} \theta+\sinh ^{4} \theta\right)+\frac{\epsilon^{2}}{2} \sinh ^{2} 2 \theta},
$$

so $u<0$ and $v>0$. In order to get the inverse Laplace transform of each component of the $\Omega$-matrix, we must seek for the solutions of the quartic equation $Q(z)=0$. As it is well known, a general quartic equation is a fourth-order polynomial equation of the form

$$
z^{4}+a_{3} z^{3}+a_{2} z^{2}+a_{1} z+a_{0}=0
$$

Using the familiar algebraic technique, ${ }^{48}$ it is easy to show that the roots of Eq. (A20) are given by

$$
\begin{aligned}
& z_{1}=-\frac{1}{4} a_{3}+\frac{1}{2} R+\frac{1}{2} D, \\
& z_{2}=-\frac{1}{4} a_{3}+\frac{1}{2} R-\frac{1}{2} D, \\
& z_{3}=-\frac{1}{4} a_{3}-\frac{1}{2} R+\frac{1}{2} E, \\
& z_{4}=-\frac{1}{4} a_{3}-\frac{1}{2} R-\frac{1}{2} E,
\end{aligned}
$$

where

$$
\begin{gathered}
R \equiv\left(\frac{1}{4} a_{3}^{2}-a_{2}+y_{1}\right)^{1 / 2}, \\
D \equiv\left\{\begin{array}{l}
(F(R)+G)^{1 / 2} \text { for } R \neq 0 \\
(F(0)+H)^{1 / 2} \text { for } R=0,
\end{array}\right. \\
E \equiv \begin{cases}(F(R)-G)^{1 / 2} & \text { for } R \neq 0 \\
(F(0)-H)^{1 / 2} & \text { for } R=0,\end{cases} \\
F(R) \equiv \frac{3}{4} a_{3}^{2}-R^{2}-2 a_{2}, \\
H \equiv 2\left(y_{1}^{2}-4 a_{0}\right)^{1 / 2}, \\
G \equiv \frac{1}{4}\left(4 a_{3} a_{2}-8 a_{1}-a_{3}^{3}\right) R^{-1},
\end{gathered}
$$

and $y_{1}$ is a real root of the following cubic equation:

$$
y^{3}-a_{2} y^{2}+\left(a_{1} a_{3}-4 a_{0}\right) y+\left(4 a_{2} a_{0}-a_{1}^{2}-a_{3}^{2} a_{0}\right)=0 .
$$

For convenience, let us assume that $R$, defined by Eq. (A25), does not vanish. Comparing Eqs. (A17) and (A20), we easily see that $a_{3}=2 \Lambda^{2}, a_{2}=\Lambda^{4}-u, a_{1}=-u \Lambda^{2}$, and $a_{0}=v$. Therefore, the inverse Laplace transform of $\Omega_{a b}$ is given by 


$$
\begin{aligned}
\Omega_{11}(k, \tau)= & \frac{P\left(z_{1}, t_{a}\right)}{\left(z_{1}-z_{2}\right)\left(z_{1}-z_{3}\right)\left(z_{1}-z_{4}\right)} e^{z_{1} \tau} \\
& +\frac{P\left(z_{2}, t_{a}\right)}{\left(z_{2}-z_{1}\right)\left(z_{2}-z_{3}\right)\left(z_{2}-z_{4}\right)} e^{z_{2} \tau} \\
& +\frac{P\left(z_{3}, t_{a}\right)}{\left(z_{3}-z_{1}\right)\left(z_{3}-z_{2}\right)\left(z_{3}-z_{4}\right)} e^{z_{3} \tau} \\
& +\frac{P\left(z_{4}, t_{a}\right)}{\left(z_{4}-z_{1}\right)\left(z_{4}-z_{2}\right)\left(z_{4}-z_{3}\right)} e^{z_{4} \tau}, \\
\Omega_{12}(k, \tau)= & \Omega_{21}(k, \tau)=-\left[\frac{t_{b} z_{1}+t_{b} \Lambda^{2}}{\left(z_{1}-z_{2}\right)\left(z_{1}-z_{3}\right)\left(z_{1}-z_{4}\right)} e^{z_{1} \tau}\right. \\
& +\frac{t_{b} z_{2}+t_{b} \Lambda^{2}}{\left(z_{2}-z_{1}\right)\left(z_{2}-z_{3}\right)\left(z_{2}-z_{4}\right)} e^{z_{2} \tau} \\
& +\frac{t_{b} z_{3}+t_{b} \Lambda^{2}}{\left(z_{3}-z_{1}\right)\left(z_{3}-z_{2}\right)\left(z_{3}-z_{4}\right)} e^{z_{3} \tau} \\
& \left.+\frac{t_{b} z_{4}+t_{b} \Lambda^{2}}{\left(z_{4}-z_{1}\right)\left(z_{4}-z_{2}\right)\left(z_{4}-z_{3}\right)} e^{z_{4} \tau}\right]
\end{aligned}
$$

and finally, $\Omega_{22}(k, \tau)=\Omega_{11}\left(k, \tau ; t_{a} \rightarrow t_{d}\right)$. The roots $z_{i}$ are given by

$$
\begin{aligned}
& z_{1}=-\frac{\Lambda^{2}}{2}+\frac{1}{2} i \sigma+\frac{1}{2} i \gamma, \\
& z_{2}=-\frac{\Lambda^{2}}{2}+\frac{1}{2} i \sigma-\frac{1}{2} i \gamma, \\
& z_{3}=-\frac{\Lambda^{2}}{2}-\frac{1}{2} i \sigma+\frac{1}{2} i \gamma, \\
& z_{4}=-\frac{\Lambda^{2}}{2}-\frac{1}{2} i \sigma-\frac{1}{2} i \gamma,
\end{aligned}
$$

with $\sigma=\left(|u|-y_{1}\right)^{1 / 2}$ and $\gamma=\left(-\Lambda^{4}+|u|+y_{1}\right)^{1 / 2}$ being real quantities. Equations (A32) and (A33) can be rewritten in a simpler form as

$$
\begin{aligned}
\Omega_{11}(k, \tau)= & -\left[\left(\cos \left(\frac{(\sigma+\gamma)}{2} \tau\right)+\frac{\Lambda^{2}}{(\sigma+\gamma)} \sin \left(\frac{(\sigma+\gamma)}{2} \tau\right)\right) h_{1}\right. \\
& +\left(\cos \left(\frac{(\sigma-\gamma)}{2} \tau\right)+\frac{\Lambda^{2}}{(\sigma-\gamma)} \sin \left(\frac{(\sigma-\gamma)}{2} \tau\right)\right) h_{2} \\
& +8 t_{a} \sin \left(\frac{\sigma \tau}{2}\right) \sin \left(\frac{\gamma \tau}{2}\right) \\
& \left.+4 t_{a} \Lambda^{2}\left(g_{1} \sin \left(\frac{(\sigma+\gamma)}{2} \tau\right)+g_{2} \sin \left(\frac{(\sigma-\gamma)}{2} \tau\right)\right)\right] \frac{e^{\left(-\Lambda^{2} / 2\right) \tau}}{8 \sigma \gamma}
\end{aligned}
$$




$$
\begin{aligned}
\Omega_{12}(k, \tau)= & \Omega_{21}(k, \tau)=\frac{t_{b}}{2 \sigma \gamma}\left[\frac{\Lambda^{2}}{(\sigma+\gamma)} \sin \left(\frac{(\sigma+\gamma)}{2} \tau\right)-\frac{\Lambda^{2}}{(\sigma-\gamma)} \sin \left(\frac{(\sigma-\gamma)}{2} \tau\right)+\right. \\
& \left.-2 \sin \left(\frac{\sigma \tau}{2}\right) \sin \left(\frac{\gamma \tau}{2}\right)\right] e^{\left(-\Lambda^{2} / 2\right) \tau},
\end{aligned}
$$

where

$$
\begin{gathered}
h_{1}=-(\sigma+\gamma)^{2}-\Lambda^{4}, \\
h_{2}=(\sigma-\gamma)^{2}+\Lambda^{4}, \\
g_{1}=i-\frac{2 \Lambda^{2}}{(\sigma+\gamma)}, \\
g_{2}=i-\frac{2 \Lambda^{2}}{(\sigma-\gamma)},
\end{gathered}
$$

and, as before, $\Omega_{22}(k, \tau)=\Omega_{11}\left(k, \tau ; t_{a} \rightarrow t_{d}\right)$. Let us consider the convergence of the stochastic process. In order for our stochastic process to converge, the retarded Green's function for the diffusion problem should obey $\left.G_{a b}(k, \tau)\right|_{\tau \rightarrow \infty} \rightarrow 0$. In other words, we must have $\left.\Omega_{a b}(k, \tau)\right|_{\tau \rightarrow \infty}$ $\rightarrow 0$. From the above expressions, it is easy to see that the stochastic process will converge if all roots of the polynomial $Q(z)$ are negative. Indeed, from the usual relations between the zeros of a polynomial and the coefficients of the polynomial equation, we get $\Pi_{i} z_{i}=a_{0}=v>0$. So, we have an even combination of equal signs in the roots. In other words, it is possible to obtain four negative roots as long as we have $\sqrt{-|u|+y_{1}}+\sqrt{\Lambda^{4}-|u|-y_{1}}<\Lambda^{2}$. Another way to reach convergence is to demand that all roots are complex and set each real part of the roots to be negative. Since, in our case, all the real parts are equal and negative, we have chosen to follow this way. So, we will have convergence if the quantities $\sigma$ and $\gamma$ are real, as imposed before. This leads us to the following conditions: $|u|-y_{1}>0$ and $|u|+y_{1}-\Lambda^{4}>0$, or, combining those requirements, $|u|>\max \left\{y_{1}, \Lambda^{4}\right.$ $\left.-y_{1}\right\}$.

Now let us present the quantity $y_{1}$. As was stated before, $y_{1}$ is a real root of a cubic equation

$$
z^{3}+b_{2} z^{2}+b_{1} z+b_{0}=0
$$

Comparing Eqs. (A17), (A20), (A31), and (A44), we have the following identifications: $b_{2}=u$ $-\Lambda^{4}, b_{1}=-2 \Lambda^{4} u-4 v$, and $b_{0}=-4 u v-u^{2} \Lambda^{4}$. If we let

$$
\begin{gathered}
q=\frac{1}{3} b_{1}-\frac{1}{9} b_{2}^{2}, \\
r=\frac{1}{6}\left(b_{1} b_{2}-3 b_{0}\right)-\frac{1}{27} b_{2}^{3},
\end{gathered}
$$

we will have that

$$
\begin{gathered}
q=-\frac{4}{9} \Lambda^{4} u-\frac{\Lambda^{8}}{9}-\frac{4}{3} v-\frac{u^{2}}{9}, \\
r=\frac{4}{9} \Lambda^{8} u+\frac{2}{3} \Lambda^{4} v+\frac{1}{18} \Lambda^{4} u^{2}+\frac{4}{3} u v-\frac{1}{27} \Lambda^{12}+\frac{1}{27} u^{3} .
\end{gathered}
$$

So, writing $s_{1}=\left(r+\sqrt{q^{3}+r^{2}}\right)^{1 / 3}$ and $s_{2}=\left(r-\sqrt{q^{3}+r^{2}}\right)^{1 / 3}$, we have that 


$$
y_{1}=\left(s_{1}+s_{2}\right)+\frac{\Lambda^{4}-u}{3} .
$$

As one can see, $y_{1}>0$. Also, in the limit $\epsilon \rightarrow 0, y_{1}$ becomes a polynomial of $\Lambda$.

${ }^{1}$ T. Matsubara, Prog. Theor. Phys. 14, 351 (1955).

${ }^{2}$ A. A. Abrikosov, L. Gorkov, and I. Dzyaloshinski, Method of Quantum Field Theory in Statistical Physics (Pergamon, Oxford, 1967).

${ }^{3}$ J. Schwinger, J. Math. Phys. 2, 407 (1961).

${ }^{4}$ L. V. Keldysh, Sov. Phys. JETP 20, 235 (1964).

${ }^{5}$ L. Leplae, H. Umezawa, and F. Mancini, Phys. Rep. 10, 151 (1974).

${ }^{6}$ H. Matsumoto, Fortschr. Phys. 25, 1 (1977).

${ }^{7}$ A. A. Abrikosov, L. Gorkov, and I. Dzyaloshinski, Sov. Phys. JETP 9, 636 (1959).

${ }^{8}$ Proceedings of the Workshop on Thermal Quantum Field Theories and Their Applications, edited by K. L. Kowalsky, N. P. Landsman, and Ch. G. van Weert (Case-Western Reserve University, Cleveland, Ohio, 1988) [ Physica A 158, 1 (1989)].

${ }^{9}$ G. Menezes and N. F. Svaiter, Physica A 374, 617 (2007).

${ }^{10}$ R. F. Fox, J. Stat. Phys. 16, 259 (1977).

${ }^{11}$ R. F. Fox, J. Math. Phys. 18, 2331 (1977).

${ }^{12}$ R. Zwanzig, Non-Equilibrium Statistical Mechanics (Oxford University Press, New York, 2001).

${ }^{13}$ R. Kubo, M. Toda, and N. Hashitsume, Statistical Physics (Springer, Heidelberg, 1991).

${ }^{14}$ G. Parisi and Y. S. Wu, Sci. Sin. 24, 483 (1981).

${ }^{15}$ B. Sakita, Quantum Theory of Many Variable Systems and Fields (World Scientific, Singapore, 1985).

${ }^{16}$ P. Damgaard and H. Hüffel, Stochastic Quantization (World Scientific, Singapore, 1988).

${ }^{17}$ M. Namiki, Prog. Theor. Phys. Suppl. 111, 1 (1993).

${ }^{18}$ P. Damgaard and H. Hüffel, Phys. Rep. 152, 227 (1987).

${ }^{19}$ H. Hüffel and H. Rumpf, Phys. Lett. 148B, 104 (1984).

${ }^{20}$ E. Gozzi, Phys. Lett. 150B, 119 (1985).

${ }^{21}$ H. Hüffel and P. V. Landshoff, Nucl. Phys. B 260, 545 (1985).

${ }^{22}$ D. J. E. Callaway, F. Cooper, J. R. Klauder, and H. A. Rose, Nucl. Phys. B 262, 19 (1985).

${ }^{23}$ T. Fukai, H. Nakazato, J. Ohba, K. Okano, and Y. Yamanaka, Prog. Theor. Phys. 69, 1600 (1983).

${ }^{24}$ P. H. Damgaard and K. Tsokos, Nucl. Phys. B 235, 75 (1984).

${ }^{25}$ P. H. Damgaard, Prog. Theor. Phys. Suppl. 111, 43 (1993).

${ }^{26}$ G. Parisi, Phys. Lett. B 131, 393 (1983).

${ }^{27}$ J. R. Klauder and W. P. Peterson, J. Stat. Phys. 39, 53 (1985).

${ }^{28}$ J. R. Klauder, Phys. Rev. A 29, 2036 (1984).

${ }^{29}$ J. Ambjorn and S. K. Yang, Phys. Lett. 165, 140 (1985).

${ }^{30}$ H. Okamoto, K. Okano, L. Schülke, and S. Tanaka, Nucl. Phys. B 324, 684 (1989).

${ }^{31}$ G. Guralnik and C. Pehlevan, Nucl. Phys. B 822, 349 (2009).

${ }^{32}$ G. Guralnik and C. Pehlevan, Nucl. Phys. B 811, 519 (2009).

${ }^{33}$ G. Menezes and N. F. Svaiter, J. Math. Phys. 47, 073507 (2006).

${ }^{34}$ G. Menezes and N. F. Svaiter, J. Math. Phys. 49, 102301 (2008).

${ }^{35}$ J. Berges and I.-O. Stamatescu, Phys. Rev. Lett. 95, 202003 (2005).

${ }^{36}$ J. Berges, Sz. Borsányi, D. Sexty, and I.-O. Stamatescu, Phys. Rev. D 75, 045007 (2007).

${ }^{37}$ M. Falcioni, E. Marinari, M. L. Paciello, G. Parisi, B. Tagliente, and Y. S. Zhang, Nucl. Phys. B 215, 265 (1983).

${ }^{38}$ G. Aarts, Phys. Rev. Lett. 102, 131601 (2009).

${ }^{39}$ J. Smit, Introduction to Quantum Field Theory on a Lattice (Cambridge University Press, Cambridge, 2002).

${ }^{40}$ M. Creutz, Quarks, Gluons and Lattices (Cambridge University Press, Cambridge, 1983).

${ }^{41}$ M. Le Bellac, Thermal Field Theory (Cambridge University Press, Cambridge, 1996).

${ }^{42}$ A. J. Niemi and G. W. Semenoff, Nucl. Phys. B 230, 181 (1984).

${ }^{43}$ N. P. Landsman and Ch. G. van Weert, Phys. Rep. 145, 141 (1987).

${ }^{44}$ E. Floratos and J. Iliopoulos, Nucl. Phys. B 214, 392 (1983).

${ }^{45}$ H. Nakazato and Y. Yamanaka, Phys. Rev. D 34, 492 (1986).

${ }^{46}$ G. Menezes and N. F. Svaiter, J. Phys. A: Math. Theor. 40, 8545 (2007).

${ }^{47}$ T. C. de Aguiar, G. Menezes, and N. F. Svaiter, Class. Quantum Grav. 26, 075003 (2009).

${ }^{48}$ M. Abramowitz and I. A. Stegun, Handbook of Mathematical Functions with Formulas, Graphs, and Mathematical Tables (Dover, New York, 1972). 